\title{
Approximate Theoretical Calculation of Continuum Opacities
}

\author{
S. S. Penner* and M. Thomas $\dagger$ \\ California Institute of Technology, Pasadena, Calif.
}

\begin{abstract}
An approximate procedure is described for the theoretical calculation of the spectral absorption coefficient produced by bound-free and free-free transitions in plasmas containing polyelectronic atoms and ions. Our method of calculation is based on the assumption that only two ionized species make important contributions to the opacity and that these two ionic constituents are present in equal concentrations. The approximate formulas are shown to yield results that are in good accord with estimates based on detailed numerical computations for nitrogen.
\end{abstract}

\section{Introduction}

$\mathbf{A}$ PPROXIMATE expressions for the Rosseland and Planck mean free paths associated with the continuum radiation in ionized plasmas have been derived by Menzel and Pekeris ${ }^{1}$ and by Pappert and Penner. ${ }^{2}$ The last named authors followed a procedure described by Raizer.3. 4 The method of calculation introduced by Raizer, ${ }^{3}$ and used by Pappert and Penner $^{2}$ differs from that employed in the earlier work of Menzel and Pekeris ${ }^{1}$ by including only two representative $m$ times ionized atoms instead of approximating the sum over the ions by an integral. The physical reasons for the use of this approximation may be made plausible by referring to the schematic diagram shown in Fig. 1 where the concentration of $m$ ions (i. e., of $m$ times ionized atoms) is shown as a function of temperature. We note that there will be temperatures $T$ at which the plasma composition is well described by the presence of two ionic constituents that are present in equal concentration. Since all physical observables are expected to vary continuously with temperature, we expect that the use of the assumption that only two $m$ ions are present in equal concentrations for all values of $T$ must lead to a good prediction of electron concentration at all temperatures. The use of this idea, together with the introduction of several supplementary simplifications due to Raizer, has previously ${ }^{2}$ been shown to lead to results that agree, within about a factor of 2 , with the best available numericals computations for Rosseland and Planck mean free paths. On the other hand, it is $\mathrm{known}^{8}$ that the errors introduced by neglecting line radiation are of the same magnitude as, or larger than, the previously noted discrepancies resulting from the use of highly simplified computational procedures, particularly at elevated temperatures and low densities. For this reason, it appears justified to avoid the labor involved in obtaining a complete solution to the Saha equation and to utilize again the approximation of two $m$ ions present in equal concentrations. We shall then show that the resulting estimates for the spectral absorption coefficients of nitrogen also agree, well

Presented as Preprint 64-58 at the AIAA Aerospace Sciences Meeting, New York, January 20-22, 1964. This work was supported by the Office of Naval Research under Contract Nonr$220(45)$, NR 015 401. A preliminary account of the studies described in this paper was given at the Mollier Colloquium of the Verband Deutscher Ingenieure at Konstantz, West Germany, on October 25, 1963.

* Formerly Professor of Jet Propulsion at the Kármán Laboratory of Fluid Mechanics and Jet Propulsion, Daniel and Florence Guggenheim Jet Propulsion Center; now Professor and Chairman, Department of the Aerospace and Mechanical Engineering Sciences, University of California at San Diego, La Jolla, Calif. Associate Fellow Member AIAA.

† Institute Scholar, Research Assistant, Kármán Laboratory of Fluid Mechanics and Jet Propulsion, Daniel and Florence Guggenheim Jet Propulsion Center. Student Member AIAA.

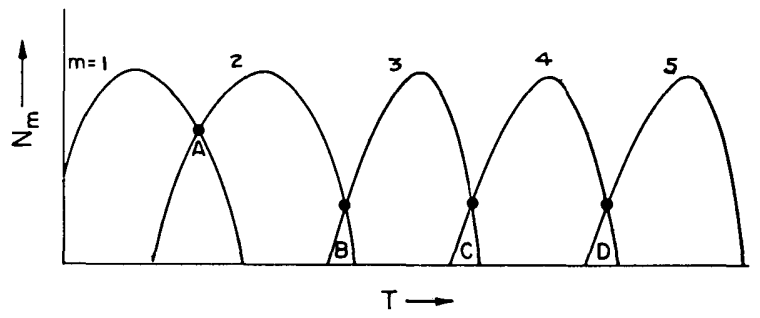

Fig. 1 Schematic diagram showing the concentration of $m$ ions, $N_{m}$, as a function of temperature $T$. At the points $A, B, C, D$, etc., the plasma composition is well described by the presence of two $m$ ions that are present in equal concentrations.

within a factor of 2 over most of the frequency range, with the best available numerical computations. $\ddagger$

\section{Theoretical Considerations}

In the following discussion we shall deviate from the analysis of Pappert and Penner ${ }^{2}$ by considering the corrections required through proper introduction of statistical weight factors, which were not treated in an entirely consistent manner by Raizer. ${ }^{3,4}$

\section{A. Degree of Ionization and Planck and Rosseland Mean Free Paths}

The Saha equation for equilibrium between the concentrations $N_{m}$ and $N_{m+1}$ of $m$ and $(m+1)$ ions, $\S$ respectively, may be written in the form

$$
\frac{N_{m+1}}{N_{m}} N_{e}=A^{\prime}\left(\frac{g_{e} g_{m+1}}{g_{m}}\right) \theta^{3 / 2} \exp \left(-\frac{I_{m}}{\theta}\right)
$$

\footnotetext{
$\ddagger$ For an exhaustive review of the very extensive literature on experimental and theoretical studies of continuum radiation, particularly on polyelectronic atoms and ions for which the hydrogenic approximation yields poor estimates, reference should be made to the review article by Finkelnburg and Peters. ${ }^{9}$ Finkelnburg and Peters cite 496 references, many of which correspond to papers published during the period 1937 to 1956, following completion of Finkelnburg's monograph on continuum spectra. ${ }^{10}$ The latter publication includes reference to 1700 papers appearing up to 1937 and beginning essentially with the classical analysis of Kramers. ${ }^{11}$ Proper appreciation of this rich historical background will serve to place the present paper in its correct perspective: a simplified prescription of calculating useful data that should be of value to the applied scientist who may content himself with approximate results that can really be improved significantly only at the expense of considerable complication in analytical procedure.

$\S$ An $m$ ion is an $m$ times ionized atom.
} 


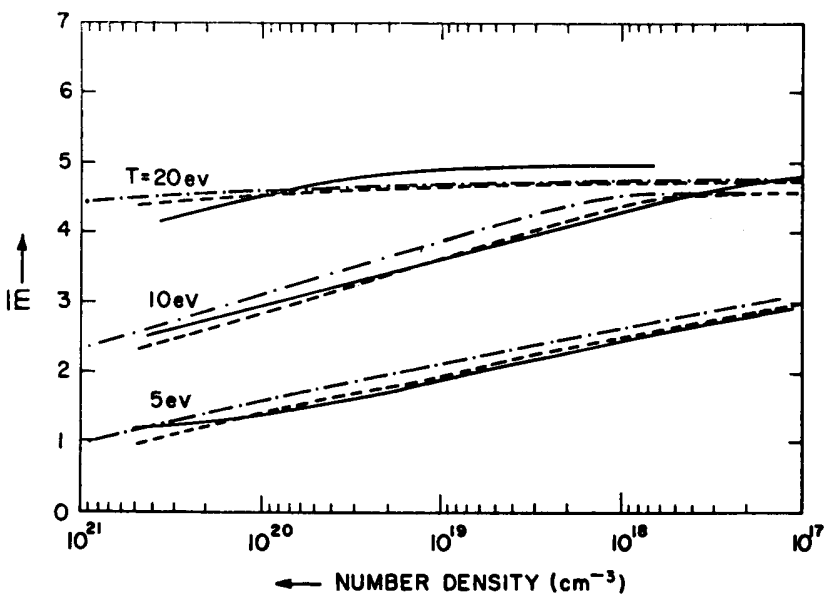

Fig. 2 The average number of electrons per atom $(\bar{m})$ as a function of the number density $N\left(\mathrm{~cm}^{-3}\right)$ at various temperatures. The dashed curve refers to results derived from Eq. (1) with $g_{e} g_{m+1} / g_{m}=1$; the dot-dash curve (which has been given previously) refers to $g_{e} g_{m+1} / g_{m}=2$; the solid curve is based on Armstrong's data.

where $N_{e}$ denotes the electron concentration;

$$
A^{\prime}=\left[\frac{2 \pi m_{e}\left(1.6 \times 10^{-12}\right)}{h^{2}}\right]^{3 / 2} \simeq 3.0 \times 10^{21} \mathrm{~cm}^{-3}-\mathrm{ev}^{-3 / 2}
$$

( $m_{e}=$ electron mass, $h=$ Planck's constant); $g_{e}, g_{m+1}$, and $g_{m}$ are the statistical weights for the electron, the $(m+1)$ ion and the $m$ ion, respectively; $\theta$ represents the energy-equivalent of the temperature in electron volts (in this paper, all energies are expressed in electron volts); and $I_{m}$ denotes the ionization potential of the $m$ ion. Raizer ${ }^{3,4}$ and Pappert and Penner ${ }^{2}$ set $g_{e}=2$ and $g_{m+1} / g_{m}=1$, whereas they used the Unsöld approximation $g_{e} g_{m+1} / g_{m}=1$ in the opacity formulas. Actually the use of the assumption $g_{e} g_{m+1} / g_{m}=1$ leads to a somewhat more satisfactory prediction of the average degree of ionization $\bar{m}$ than the statement $g_{e} g_{m+1} / g_{m}=2$, as may be seen by reference to Fig. 2 . In any case, a consistent approximation procedure requires the use of the same estimates for $g_{e} g_{m+1} / g_{m}$ everywhere.

If we employ the assumption $g_{e} g_{m+1} / g_{m}=1$ everywhere, then the values of $A=2 A^{\prime}$ appearing in the formulas of Pappert and Penner ${ }^{2}$ for the Rosseland and Planck mean free

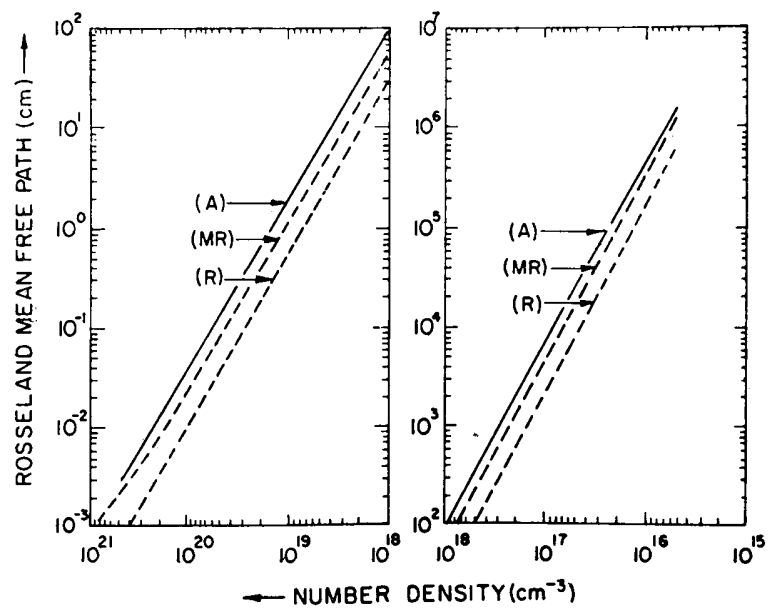

Fig. 3 The Rosseland mean free path (cm) as a function of number density $\left(\mathrm{cm}^{-3}\right)$ for nitrogen at $5 \mathrm{ev}$. The symbols A, R, and MR identify results obtained by Armstrong (A), and derived from the Raizer (R), and modified Raizer (MR) methods of Pappert and Penner, respectively. In the $R$ and MR plots, we have used consistently the approximation $g_{e} g_{m+1} / g_{m}=1$. The theoretical formulas for the $R$ and (MR) plots are given in Ref. 2.

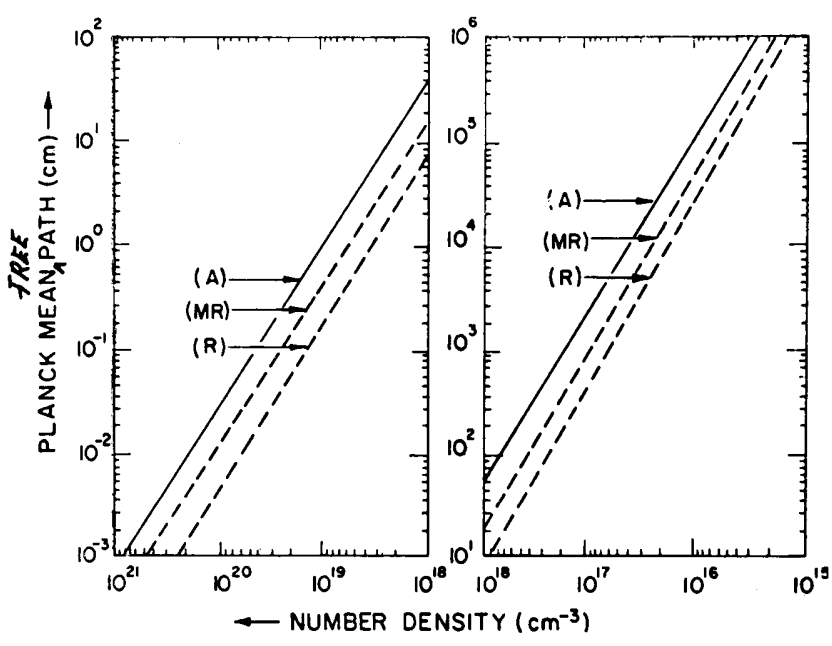

Fig. 4 The Planck mean free path (cm) as a function of number density $\left(\mathrm{cm}^{-3}\right)$ for nitrogen at $5 \mathrm{ev}$. Same symbols as Fig. 3.

paths should be divided by the factor 2 and, accordingly, all of the mean free path estimates should also be divided by a factor of 2. Representative results plotted in Fig. 3 show that the data, obtained by introducing the specified change into the modified Raizer (MR) method of Ref. 2 for the Rosseland mean free path, still agree well with Armstrong's results and that the approximate computations tend to be somewhat too low. However, with $A=2 A^{\prime}$ replaced by $A^{\prime}$, the MR method leads to a larger deviation from Armstrong's results for the Planck mean free path as may be seen by comparing Fig. 4 and the corresponding plots in Ref. 2 .

\section{B. Theoretical Calculation of Spectral Absorption Coefficients}

The following expression may be derived readily for the total linear absorption coefficient $\varkappa_{m}(x)$ associated with the bound-free contribution for an $m$ ion and the free-free con. tribution from an $(m+1)$ icn:

$\varkappa_{m}(x)=a\left(g_{e} g_{m+1} / g_{m}\right) N_{m}(m+1)^{2} \theta^{-2} \exp \left(-x_{1 m}\right) F_{m}(x)$

where

$$
\begin{gathered}
a=\frac{16 \pi^{2}}{3(3)^{1 / 2}} \frac{e^{6}}{c h}\left(1.6 \times 10^{-12}\right)^{-2} \simeq 7.3 \times 10^{-16} \mathrm{~cm}^{2}-(e v)^{2} \\
x=\frac{h \nu}{\theta} \quad x_{1 m}=\frac{I_{m}}{\theta}
\end{gathered}
$$

Here $N_{m}$ is the number density $\left(\mathrm{cm}^{-3}\right)$ of $m$ ions in the ground state. $\pi$ For atoms and ions more complicated than those treated here, the labor involved is increased because of the necessity of estimating the ionization potentials of the contributing ions. Since the hydrogenic approximation has been made for the excited states, the frequency-dependent factor is given by

$$
F_{m}(x)=x^{-3}\left[2 x_{1 m} \Sigma n^{-3} \exp \left(x_{1 m} / n^{2}\right)+1\right]
$$

The first term in the brackets represents the bound-free contribution, whereas the second term represents the free-free contribution to the absorption coefficient. In an ionized gas containing $m$ ions with $m=0,1,2 \ldots$, the total continuum absorption coefficient is finally obtained by summing the contributions made by the separate $m$ ions. Thus,

$$
\begin{array}{r}
\varkappa^{\prime}(x)=\frac{\varkappa(x)}{1-\exp (-x)}=a \theta^{-2} \Sigma_{m} N_{m}\left(\frac{g_{e} g_{m+1}}{g_{m}}\right)(m+1)^{2} \times \\
\exp \left(-x_{1 m}\right) F_{m}(x)
\end{array}
$$

I For all practical purposes, $N_{m}$ equals the total number of $m$ ions per unit volume. 
where $\mathcal{K}^{\prime}(x)$ denotes the spectral absorption coefficient with the induced emission term excluded.

Menzel and Pekeris ${ }^{1}$ and Raizer ${ }^{4}$ have performed an approximate evaluation for the radiation mean free paths by utilizing the fact that configuration splitting of the $2 n^{2}$ degenerate hydrogenic levels makes plausible the replacement
Furthermore, since

$$
N_{m+(1 / 2)}^{-}=N_{m-(1 / 2)}^{-}=N / 2
$$

only the two terms $m=\bar{m}-\left(\frac{3}{2}\right)$ and $m=\bar{m}-\left(\frac{1}{2}\right)$ contribute to the sums in the first of the Eqs. (10) and in Eq. (13). We find the following results for the sums occurring in Eqs. (10):

$$
\left.\begin{array}{rl}
\Sigma_{m} N_{m}\left(g_{e} g_{m+1} / g_{m}\right)(m+1)^{2} \exp \left(-x_{1 m}\right) & =\left(N \bar{m} / A^{\prime} \theta^{3 / 2}\right) \Sigma_{m} N_{m+1}(m+1)^{2} \\
& \simeq\left(N^{2} \bar{m} / A^{\prime} \theta^{3 / 2}\right)\left(\bar{m}^{2}+\frac{1}{4}\right) \text { for } x<x_{1 m_{1}} \\
& \simeq\left(N^{2} \bar{m} / 2 A^{\prime} \theta^{3 / 2}\right)\left(\bar{m}+\frac{1}{2}\right)^{2} \text { for } x_{1 m_{1}}<x<x_{1 m_{2}}
\end{array}\right\}
$$

of the sum over $n$, which occurs in Eqs. (4) and (5), by an integration, i.e.,

$$
\Sigma \frac{2 x_{1 m}}{n^{3}} \exp \left(\frac{x_{1 m}}{n^{2}}\right) \rightarrow \int_{0}^{x} e^{y} d y=e^{x}-1 \quad x<x_{1 m}
$$

whence

$$
F_{m}(x) \simeq x^{-3} e^{x} \text { for } \quad x<x_{1 m}
$$

Here $N$ is the total number density per cubic centimeter and $m_{1}=\bar{m}-\left(\frac{1}{2}\right), m_{2}=\bar{m}+\left(\frac{1}{2}\right)$, and we have made the assumption $g_{e} g_{m+1} / g_{m}=1$ in Eq. (16). It should be noted that $x<x_{1 m_{1}}$ corresponds physically to $h \nu<I_{m-(1 / 2)}, x_{1 m_{1}}<x<$ $x_{1 m_{2}}$ to $I_{m}^{-}-(1 / 2)<h \nu<I_{m+(1 / 2)}^{-}$, and $x>x_{1 m_{2}}$ to $h \nu>I_{m+1}^{-1 / 2)}$. Thus we obtain the following expressions for the spectral absorption coefficient:

$$
\varkappa^{\prime}(x)=\frac{a}{\theta^{2} x^{3}}\left\{\begin{array}{l}
\left(N^{2} \bar{m} / A^{\prime} \theta^{3 / 2}\right)\left(\bar{m}^{2}+\frac{1}{4}\right)[\exp (x)] \text { for } x<x_{1 m_{1}} \\
\left\{\left(\Lambda^{2} \bar{m} / 2 A^{\prime} \theta^{3 / 2}\right)\left(\bar{m}+\frac{1}{2}\right)^{2}[\exp (x)]+N\left(\bar{m}+\frac{1}{2}\right)^{2} x_{1 m_{1}}\right\} \text { for } x_{1 m_{1}}<x<x_{1 m_{2}} \\
N\left[\left(\bar{m}+\frac{1}{2}\right)^{2} x_{1 m_{1}}+\left(\bar{m}+\frac{3}{2}\right)^{2} x_{1 m_{2}}\right] \text { for } x>x_{1 m_{2}}
\end{array}\right.
$$

Thus, for the case in which the energy of the incident photon is less than the ionization potential of the $m$ ion, the frequency dependent factor $F_{m}(x)$ is a universal function of $x$.

For $x>x_{1 m}$, it is assumed that the dominant role is played by the ground level $n=1$. Therefore,

$$
F_{m}(x)=2 x_{1 m} x^{-3} \exp \left(x_{1 m}\right) \quad x>x_{1 m}
$$

In view of Eqs. (7) and (8), we may use the following approximate representation for $F_{m}(x)$ :

$$
F_{m}(x) \simeq\left\{\begin{array}{l}
{\left[\exp (x) / x^{3}\right] \text { for } x<x_{1 m}} \\
\left(2 x_{1 m} / x^{3}\right) \exp \left(x_{1 m}\right) \text { for } x \geq x_{1 m}
\end{array}\right.
$$

Hence,

$$
\varkappa^{\prime}(x)=\left(a / \theta^{2} x^{3}\right)\left\{\begin{array}{c}
{[\exp (x)] \Sigma_{m} N_{m} \times\left(g_{e} g_{m+1} / g_{m}\right)(m+1)^{2} \times} \\
\exp \left(-x_{1 m}\right) \text { for } x<x_{1 m} \\
2 \Sigma_{m} N_{m}\left(g_{e} g_{m+1} / g_{m}\right)(m+1)^{2} x_{1 m} \\
\text { for } x \geq x_{1 m}
\end{array}\right.
$$

In order to obtain an explicit relation for $\mathcal{\varkappa}^{\prime}(x)$, it is now necessary to evaluate the sums over the $m$ ions. A rigorous evaluation of these sums requires the determination of $N_{m}$ with proper allowance for each of the equilibria

$$
N_{m} \rightleftarrows N_{m+1}+e^{-}
$$

Instead of solving the complete set of simultaneous Saha equations, we estimate the average degree of ionization $\bar{m}$ from Eq. (1) written in the form

$$
I_{\vec{m}-(1 /}=\theta \ln \left\{\frac{A^{\prime}\left[g_{e} g_{m}^{-}+(1 / 2) / g_{m}^{-}-(1 / 2)\right] \theta^{3 / 2}}{N \bar{m}}\right\}
$$

accord with our earlier discussion and with the method of Pappert and Penner, ${ }^{2}$ we regard Eq. (12) to be consistent with the Saha equation in the sense that the hypothetical ion species corresponding to $m=\bar{m}+\left(\frac{1}{2}\right)$ and $m=\bar{m}-\left(\frac{1}{2}\right)$ are equal in number. Using Eq. (1), it is now possible to rewrite one of the sums occurring in Eq. (10) as

$$
\begin{aligned}
& \Sigma_{m} N_{m}\left(g_{e} g_{m+1} / g_{m}\right)(m+1)^{2} \exp \left(-x_{1 m}\right)= \\
&\left(N \bar{m} / A^{\prime} \theta^{3 / 2}\right) \Sigma_{m} N_{m+1}(m+1)^{2}
\end{aligned}
$$

The assumption $g_{e} g_{m+1} / g_{m}=1$ affects only the terms in Eq. (17) which do not contain $A^{\prime}$. Actual calculations of this ratio for polyelectronic atoms may be performed easily for the ground electronic state and indicate that $g_{\varepsilon} g_{m+1} / g_{m}$ may be smaller or larger than unity by as much as factors of about $\frac{1}{3}$ to about 3 and that an average value of unity is not an unreasonable choice.

In order to complete the semianalytical representation for $\varkappa^{\prime}(x)$, it is now necessary to specify $\bar{m}$ in terms of $\theta$ and $N$. An implicit representation for $\bar{m}$ is given through the Raizer approximation specified in Eq. (12). Equations (17) and (12) permit ready calculation of $\mathcal{\varkappa}^{\prime}(x)$ to the hydrogenic (modified Raizer) representation for all values of $\theta$ and $N$. Reference to Eqs. (17) and (12) shows that $\boldsymbol{\kappa}^{\prime}$, for fixed values of $N$ and $\theta$, increases as $\nu^{-3} \exp (h \nu / \theta)$ until $\nu=I_{m-(1 / 2)}^{-} / h$; for $I_{m-(1 / 2)}^{-}<$ $h \nu<I_{\bar{m}+(1 / 2)}, \varkappa^{\prime}$ is given by the sum of two terms, one of which increases as $\nu^{-3} \exp (h \nu / \theta)$ and the other of which decreases as $\nu^{-3}$; finally, for $\nu>I_{m+(y / z)}^{-} / h, \varkappa^{\prime}$ becomes inversely proportional to $\nu^{3}$. This simplified form for $\varkappa^{\prime}(x)$ is the direct

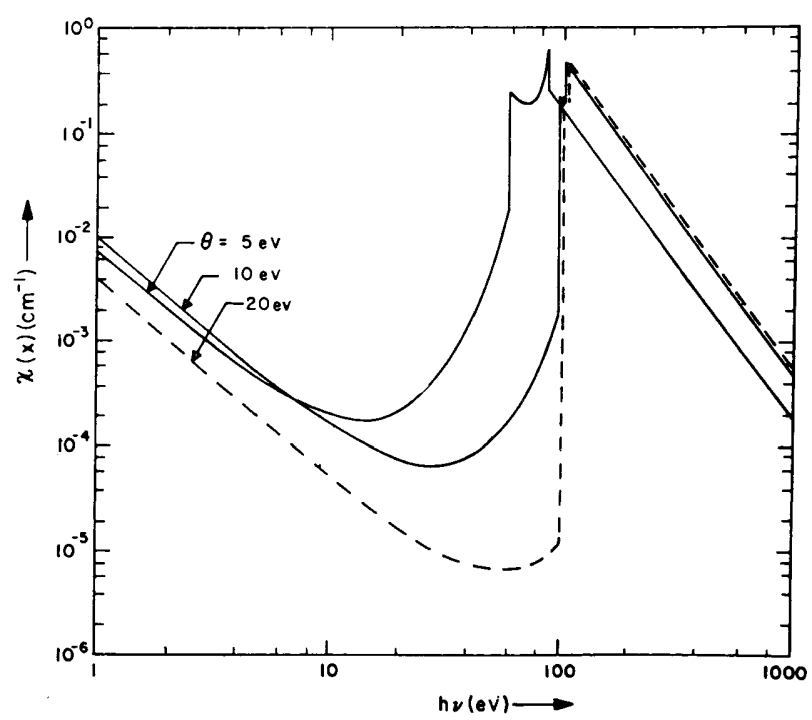

Fig. 5 A simplified representation for the spectral absorption coefficient of nitrogen as a function of frequency for $\theta=5,10$, and 20 ev for $10^{17}$ atoms $/ \mathrm{cm}^{3}$. 


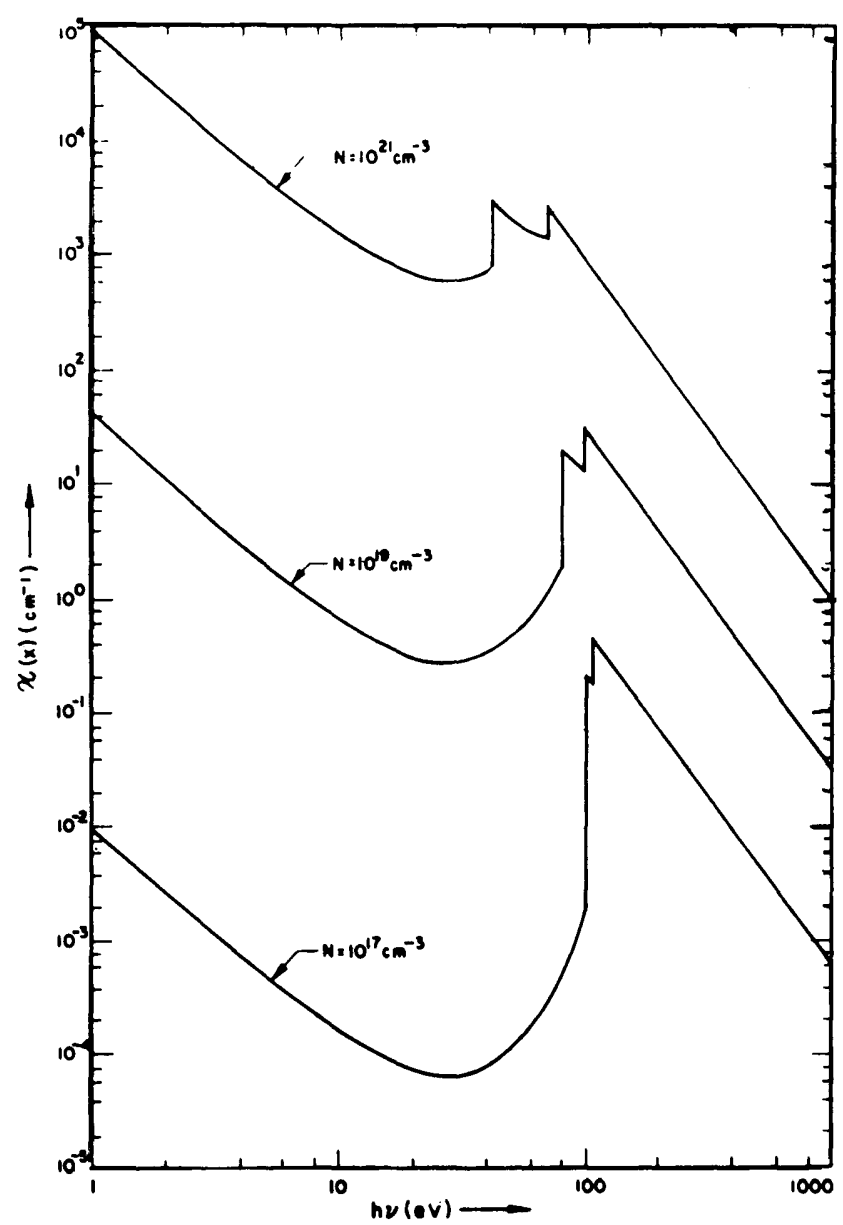

Fig. 6 A simplified representation for the spectral absorption coefficient of nitrogen at $10 \mathrm{ev}$ for $10^{17}, 10^{19}$, and $10^{21}$ atoms $/ \mathbf{c m}^{3}$.

result of the approximation that the number density of ionized atoms has been assumed to be vanishingly small except for the $m$ ions characterized by $m=\bar{m} \pm\left(\frac{1}{2}\right)$.

\section{Comparison between the Approximate and Numerical Results}

Representative plots of $\varkappa(x=h \nu / \theta)=\varkappa^{\prime}(x)[1-\exp (-x)]$, i.e., of the spectral absorption coefficient with the induced emission term included, as a function of $\nu$ are shown in Fig. 5 for nitrogen at $5 \mathrm{ev}, 10 \mathrm{ev}$, and $20 \mathrm{ev}$ for a number density of $10^{17} \mathrm{~cm}^{-3} ; \varkappa(x)$ is shown in Fig. 6 as a function of $\nu$ for $\theta=10$ ev and for number densities of $10^{17}, 10^{19}$, and $10^{21} \mathrm{~cm}^{-3}$. In constructing the plots shown in Figs. 5 and 6 , we have used the calculated values for $\bar{m}$ based on Armstrong's data. ${ }^{5}$

Figure 7 shows the values of $\varkappa^{\prime}(x)=\varkappa(x)[1-\exp (-x)]^{-1}$ calculated from Eq. (17) with values of $\bar{m}$ taken from Ref. 5 . Also shown in Fig. 7 are Armstrong's ${ }^{5}$ more accurate calculations. Armstrong did not use the method of Seaton ${ }^{6,7}$ but employed screened hydrogenic cross sections, made full allowance for LS term splitting, for the correct ionization potentials, and for an approximate plasma interaction effect.**

Reference to Fig. 7 shows that significant discrepancies occur only for photon energies that are sufficiently large to ionize the nitrogen ion in its ground state, because the hydrogenic model is then no longer a reasonable approximation and because statistical weight factors influence the results. $\dagger \dagger$ On

** The authors wish to thank B. H. Armstrong for furnishing a precise categorization of the assumptions used in his calculations.

t† As was indicated previously in this discussion, we have avoided the complications associated with the statistical weight factors in terms not containing $A^{\prime}$ by setting $g_{e} g_{m+1} / g_{m}$ arbitrarily equal to unity.

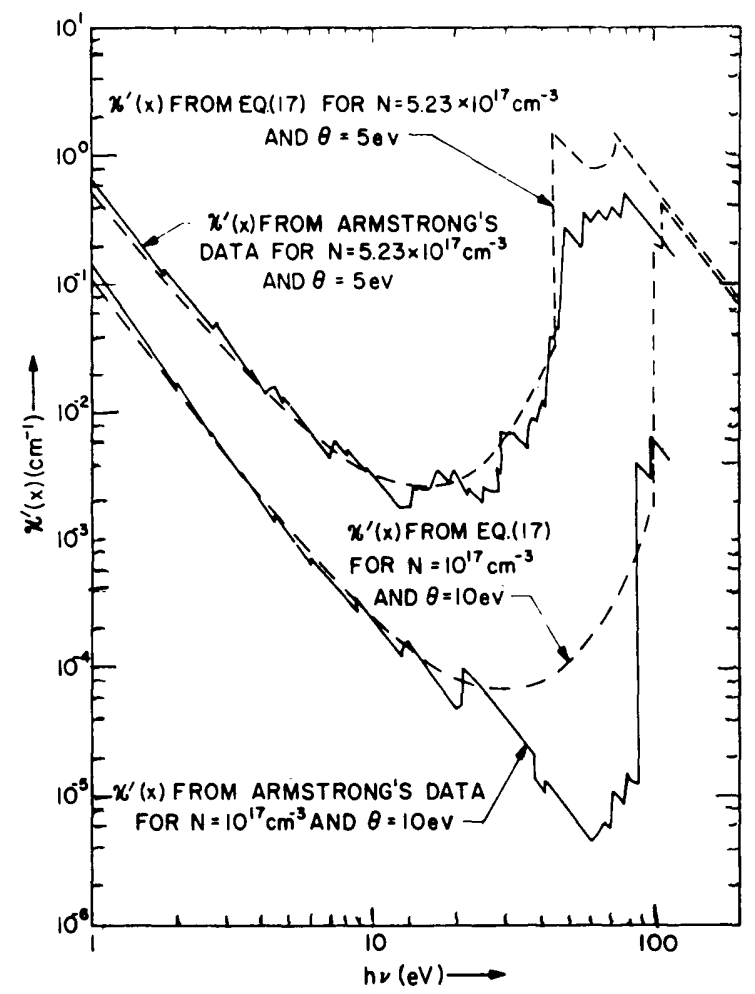

Fig. 7 The spectral absorption coefficient, without the induced emission term, for nitrogen as calculated from Eq. (17) and, using the best available methods, as calculated by Armstrong ${ }^{5}$ for $\theta=5 \mathrm{ev}, N=5.23 \times 10^{17} \mathrm{~cm}^{-3}$ and $\theta=10 \mathrm{ev}, N=10^{17} \mathrm{~cm}^{-3}$.

the other hand, over most of the spectral range, the approximate treatment provides a good representation of the spectral absorption coefficient. Hence, we conclude that the highly simplified procedure that we have employed provides an adequate representation for thermodynamic function and opacities of plasmas containing polyelectronic atoms.

\section{References}

${ }^{1}$ Menzel, D. H. and Pekeris, C. L., "Absorption coefficients and hydrogen line intensities," Monthly Notices Roy. Astron. Soc. 96, 77-111 (1935).

${ }^{2}$ Pappert, R. A. and Penner, S. S., "Approximate opacity calculations for polyelectronic atoms at high temperatures," J. Quant. Spectry. Radiative Transfer 1, 258-268 (1961).

${ }^{3}$ Raizer, Yu. P., "A simple method of calculating the degree of ionization and thermodynamic functions of a multiply ionized ideal gas," Sov. Phys.-JETP 9, 1124-1125 (1959).

${ }^{4}$ Raizer, Yu. P., "Simple method for computing the mean range of radiation in ionized gases at high temperatures," Sov. Phys.-JETP 10, 769-771 (1960).

5 Armstrong, B. H., "Mean absorption coefficients of air, nitrogen, and oxygen from $22,000^{\circ}$ to $220,000^{\circ}$," Rept. LMSD4979, Lockheed Missiles and Space Div., Palo Alto, Calif. (July 1959); also Armstrong, B. H. and Meyerott, R. E., "Absorption coefficients for high-temperature nitrogen, oxygen, and air," Phys. Fluids 3, 138-40 (1960).

'Seaton, M. J., "Thermal inelastic collision processes," Rev. Mod. Phys. 30, 979-989 (1958).

7 Zhigulev, V. N., Romishevskii, Ye. A., and Vertushkin, V. K., "Role of radiation in modern gasdynamics," Inzh. Zh. 1, 60-83 (1961); also AIAA J. 1, 1473-1485 (1963).

${ }^{8}$ Stewart, J. C., private communication, General Dynamies/ Astronautics, San Diego, Calif. (March 1963).

${ }^{9}$ Finkelnburg, W. and Peters, T., "Kontinuierliche Spektren," Handbuch der Physik: Spektroskopie II (Julius Springer-Verlag, Berlin, 1957), Vol. XXVIII, pp. 79-204.

${ }^{10}$ Finkelnburg, W., Kontinuierliche Spektren (Springer-Verlag, Berlin, 1937).

${ }^{11}$ Kramers, H. A., "On the theory of $\mathrm{x}$-ray absorption and the continuous x-ray spectrum," Phil. Mag. 46, 836 (1923). 